\title{
Draft whole-genome sequence of Brevibacterium casei strain isolated from a bloodstream infection
}

\author{
Alina Olender ${ }^{1} \cdot$ Paweł Rutyna $^{2} \cdot$ Marcin Niemcewicz $^{2} \cdot$ Agnieszka Bogut $^{1} \cdot$ Marzanna Ciesielka $^{3} \cdot$ Grzegorz Teresiński $^{3}$
}

Received: 2 August 2019 / Accepted: 28 January 2020 / Published online: 17 February 2020

(C) The Author(s) 2020

\begin{abstract}
Despite its low virulence potential and a commensal lifestyle as a member of the human skin microbiota, Brevibacterium casei has been increasingly reported as an opportunistic pathogen, especially in immunocompromised patients. Here, we present the draft genome sequence of the S51 strain isolated from a bloodstream infection. To the best of the authors' knowledge, this is the first report of the draft genome sequence of the $B$. casei strain isolated from the clinical infection. The strain was identified using phenotypic and molecular methods and subsequently sequenced using the next-generation sequencing. The draft whole genome was assembled de novo, automatically annotated by Rapid Annotations using Subsystems Technology (RAST) server and scrutinized to predict the presence of virulence, resistance, and stress response proteins. The genome size of the S51 strain was $3,743,532 \mathrm{bp}$ and an average $\mathrm{G}+\mathrm{C}$ content was $68.3 \%$. The predicted genes included 48 genes involved in resistance to antibiotics (including vancomycin, fluoroquinolones, and beta-lactams) and toxic compounds (heavy metals), 16 genes involved in invasion and intracellular resistance (Mycobacterium virulence operons), and 94 genes involved in stress response (osmotic, oxidative stress, cold and heat shock). ResFinder has indicated the presence of a beta-lactamase, and a phenotypic analysis showed resistance to penicillin. This whole-genome NGS project for the S51strain has been deposited at EMBL/GenBank under the accession no. QNGF00000000.
\end{abstract}

Keywords Brevibacterium casei $\cdot$ Infection $\cdot$ Genome $\cdot$ Sequencing

The genus Brevibacterium along with other bacterial genera, including Arcanobacterium, Arthrobacter, Cellulomonas, Cellulosimicrobium, Corynebacterium (non-diphtheriae), Curtobacterium, Dermabacter, Exiguobacterium, Helcobacillus, Janibacter, Knoellia,

\section{Repositories GenBank Accession number: QNGF00000000}

Responsible Editor: Rodrigo Galhardo.

Electronic supplementary material The online version of this article (https://doi.org/10.1007/s42770-020-00236-x) contains supplementary material, which is available to authorized users.

Alina Olender

alina.olender@umlub.pl

1 Chair and Department of Medical Microbiology, Medical University of Lublin, Lublin, Poland

2 Biological Threats Identification and Countermeasure Centre, Military Institute of Hygiene and Epidemiology, Puławy, Poland

3 Chair and Department of Forensic Medicine, Medical University of Lublin, Lublin, Poland
Leifsonia, Microbacterium, Pseudoclavibacter, and Trueperella, demonstrate morphologic and biochemical features which fit in the coryneform classification [1]. This term encompasses aerobically growing, asporogenous, non-partially acid-fast, irregularly shaped small gram-positive rods [2]. Within recent years, there have been an increasing number of case reports claiming an association of coryneform bacteria with human diseases [2].

The members of the genus Brevibacterium are non-motile, non-fastidious, chemoorganotrophic, obligately aerobic, halotolerant $(\geq 6.5 \% \mathrm{NaCl})$, and catalase positive. Typical habitats for Brevibacteria include raw milk and surfaceripened cheese; they have also been found in animal sources. Moreover, these bacteria are considered a part of the microbiota of the human skin and adjacent structures. Presently, the genus Brevibacterium includes 40 identified species [https:// www.ncbi.nlm.nih.gov/Taxonomy/Browser/wwwtax.cgi?id= 1696], with $B$. casei as the most frequently isolated species from otherwise sterile human sites [3].

Brevibacteria have been mainly involved in the etiology of infections in immunocompromised patients and those 
suffering from severe underlying diseases such as malignancies or endocarditis. Important risk factors for Brevibacterium infections include indwelling foreign materials, prosthetic heart valves, or continuous ambulatory peritoneal dialysis catheters [2-6].

In this study, we report the draft genome of B. casei S51 isolated from a bloodstream infection. This is, to the best of the authors' knowledge, the first report of the draft genome sequence of the $B$. casei strain isolated from the clinical infection.

The bacterial growth was detected in three out of four peripheral venous blood cultures (BacT/Alert system blood culture bottles [bioMérieux, France]) obtained from a 50-yearold male. The primary focus of the infection could not be identified. The empirical treatment with intravenous vancomycin was administered in the patient.

Positive cultures of the bacteremic strain were subsequently subcultured onto the Columbia agar supplemented with 5\% sheep blood (bioMérieux, France). The isolates appeared as Gram-positive, club-shaped, slightly curved rods. They produced whitish grey colonies with a distinctive cheese-like odour characteristic of the genus Brevibacterium.

Preliminary identification of the species using single colonies of each cultured bacterial isolate was performed using the API Coryne system (bioMérieux, France). The result was confirmed by the PCR amplification of the 16S rRNA gene using bacterial universal primers followed by the DNA sequencing on both strands [7]. The consensus sequence was compared using the Basic Local Alignment Search Tool (BLAST).

Antibiotic susceptibility of the strain was determined using MIC Test Strips (Liofilchem, Italy) on the Mueller-Hinton agar supplemented with $5 \%$ defibrinated horse blood and $20 \mathrm{mg} / 1 \beta$-NAD (bioMérieux, France). The tested antimicrobials included penicillin, clindamycin, imipenem, meropenem, ciprofloxacin, vancomycin, teicoplanin, tetracycline, gentamicin, chloramphenicol, and trimethoprim/sulfamethoxazole. The methodology was based on the European Committee on Antimicrobial Susceptibility Testing (EUCAST) guidelines, applying susceptibility results for Corynebacterium species.

Libraries for sequencing were prepared from genomic DNA (kit for isolation of DNA from tissue and cells, Macherey-Nagel GmbH\&Co KG, Germany). Optimal quality and concentration of extracted DNA were checked with a NanoDrop 1000 Spectrophotometer (Thermo Scientific) and Qubit 2.0 Fluorometer (Life Technologies). For library preparation, the Nextera XT DNA Library Preparation Kit (Illumina) was used according to the manufacturer's instructions. The samples were sequenced on an Illumina MiSeq $2 \times$ 300-bp paired-end format. The raw sequencing data was demultiplexed and extracted in fastq format.

Total paired-end reads generated from sequencing were quality trimmed and assembled de novo using SPAdes (3.12.0) [8,9]. Gene prediction, annotation, and RNA search were performed by Rapid Annotations using Subsystems Technology (RAST) [10].

The consensus sequence of $16 \mathrm{~S}$ gene (758 bp) was compared using BLAST and revealed 95\% query cover and 99.59\% identities to Brevibacterium casei strain DSM 20657 (Accession no. NR_041996.1). The results of the identification were used to predict the strain to whole-genome sequencing.
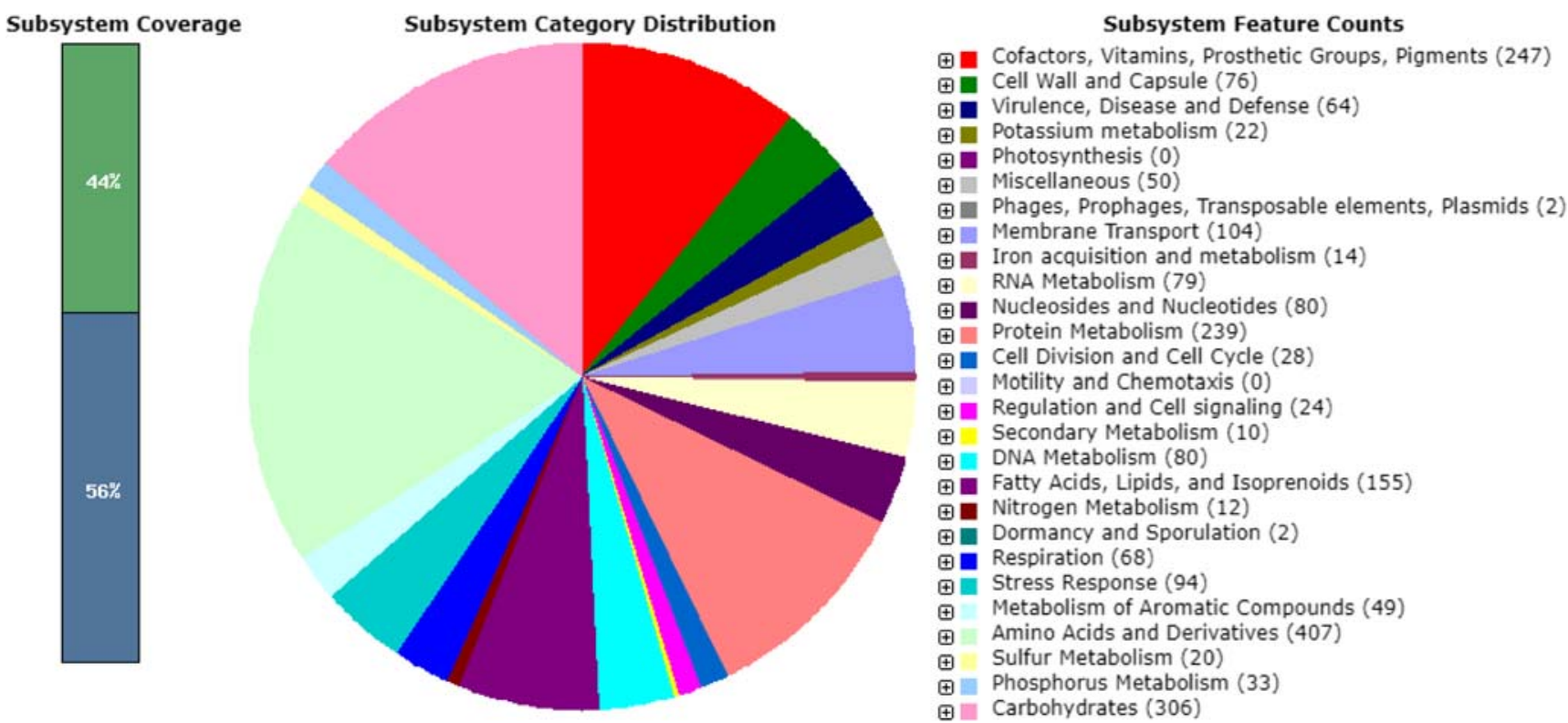

Fig. 1 An overview of the subsystem categories of the annotated draft whole-genome Brevibacterium casei S51 strain from the RAST server [10]. The pie chart demonstrates the count of each subsystem feature

and the subsystem coverage. The bar graph (on the left) determinates the ratio of coding sequences annotated in SEED subsystem features (44\%) and outside of the SEED subsystem (56\%) 
A total of 2,036,139 paired-end reads (NGS) were quality trimmed and assembled into 60 contigs with a total length of $3,743,532 \mathrm{bp}$ and an average GC content of $68.3 \%$. The $N_{50}$ of contig $B$. casei draft whole genome is $134 \mathrm{~kb}(\mathrm{~L} 50=8)$, with the longest contig being $610,939 \mathrm{~kb}$.

The automatic annotation of the obtained contigs showed the presence of 3411 open reading frames (ORFs), 3358 protein-coding sequences, and 53 RNAs.

The predicted genes included (Fig. 1):

- 48 genes involved in resistance to antibiotics and toxic compounds;

- 16 genes involved in invasion and intracellular resistance;

- 94 genes involved in stress response.

The genome of $B$. casei $\mathrm{S} 51$ contains two predicted genes involved in vancomycin resistance (vanR, van W), four genes associated with fluoroquinolone resistance ( $\operatorname{parC}$, parE, gyrA, $\operatorname{gyr} B$ ), and five genes encoding for beta-lactamases (two genes encoding for beta-lactamase EC 3.5.2.6 and single genes encoding for a beta-lactamase class $\mathrm{C}$ and other penicillinbinding proteins, metal-dependent hydrolases of the betalactamase superfamily III, and a beta-lactamase class A). The remaining resistance genes included those involved resistance to cobalt-zinc-cadmium, arsenic, chromium compounds, mercury, and copper homeostasis.

Genes involved in invasion and intracellular resistance were represented by five Mycobacterium virulence operons including those involved in SSU and LSU ribosomal protein synthesis, DNA transcription, the operon possibly involved in quinolinate biosynthesis, and the operon involved in an unknown function with a Jag protein and YidC and YidD.

Among genes involved in stress responses, 23 were associated with osmotic stress, 46 with oxidative stress, 3 with cold shock (CspA family of proteins), and 14 with heat shock (dnaK gene cluster).

Phenotypic analysis of antibiotic susceptibility of the S51 strain revealed sensitivity to all agents tested with the exception of penicillin (MIC $1.5 \mu \mathrm{g} / \mathrm{ml}$ ), chloramphenicol (MIC $24 \mu \mathrm{g} / \mathrm{ml}$ ), and trimethoprim/sulfamethoxazole (MIC $>32 \mu \mathrm{g} / \mathrm{ml}$ ).

Due to the fact that phenotypic analysis of the antibiotic resistance profile of $B$. casei S51 did not correspond to the prediction of resistance genes obtained by RAST, a more detailed investigation of resistance genes using ResFinder 3.2 [11] and CARD (Comprehensive Antibiotic Resistance Database) [12] was performed. Analysis by CARD showed loose hits for all of the predicted resistance genes.

Additional analysis trimmed *.fastq files of S51 strain by ResFinder predicted blaTEM-116 gene with $100 \%$ identity and cover responsible for beta-lactam resistance (Accession no. AY425988.1).

According to the available literature data, Brevibacteria are uncommon but important opportunistic pathogens. Starting from 1991, 15 cases of bacteremia caused by this group of bacteria have been reported $[3,5,6,13-23]$. The predominant species, responsible for 10 out of 15 cases, was $B$. casei. Single reported bacteremia cases were caused by other species including B. epidermidis [13], Brevibacterium massiliense [23] now designated as Brevibacterium ravenspurgense [24], and Brevibacterium paucivorans [6] whereas in the two remaining reports, the isolate could not be characterized to the species level. The overwhelming majority of patients reported in the publications cited above suffered from severe underlying conditions including malignancies, AIDS, Crohn's disease, pulmonary hypertension, diabetes, and chronic heart failure. Indwelling catheters could be identified as crucial risk factors for the development of Brevibacterium bacteremia in 12 patients.

It should be noted that phenotypic methods including commonly used biochemical test panels or MALDI-TOF MS technology (matrix assisted laser desorption ionization time of flight mass spectrometry) based on the analysis of the protein composition of microbial cells, may be imperfect for a definite identification of Brevibacterium species [14, 23, 24, 26]. Therefore, currently, only molecular methods such as the 16S rRNA gene sequencing enable the reliable identification

Table 1 Comparison of the genomic feature of Brevibacterium casei S51 strain with other Brevibacterium casei strains, received from the NCBI database

\begin{tabular}{llllllcr}
\hline Strain & DB accession number & Scope & Isolation source & Contigs (scaffolds) & $\begin{array}{l}\text { Genome } \\
\text { size (bp) }\end{array}$ & $\begin{array}{l}\text { OrthoANI } \\
\text { value }(\%)\end{array}$ & $\begin{array}{c}\text { isDDH value (\%) } \\
\text { S51 }\end{array}$ \\
& GCA_000314575.1 & Monoisolate & Clinical & 60 & $3,743,532$ & 100 & 100 \\
S18 & GCA_000314575.1 & Monoisolate & Human healthy skin & 43 & $3,664,641$ & 98.44 & 93.80 \\
M40 & GCA_001619685.1 & Monoisolate & Environmental & 88 & $3,769,110$ & 98.40 & 93,77 \\
UBA2623 & GCA_002339175.1 & Multispecies & Environmental & $88(59)$ & $3,687,532$ & 98.37 & 93,13 \\
UBA 7515 & GCA_002476965.1 & Metagenomes & Environmental & $310(194)$ & $3,396,412$ & 98.13 & 93.71 \\
CIP 102111 & GCA_900169275.1 & Monoisolate & Environmental & 24 & $3,840,753$ & 97.84 & 91,01 \\
OG2 & GCA_002276605.1 & Multispecies & Environmental & 315 & $3,885,661$ & 97.78 & 89.87 \\
\hline
\end{tabular}


of Brevibacteria, as reported by Asai et al. [6], Vecten et al. [23], Bernard et al. [24], and Poesen et al. [26].

In spite of an increasing role of $B$. case $i$ as an opportunistic pathogen, its genome has not as yet been sequenced. This is, to the best of the authors' knowledge, the first report of the draft genome sequence of the $B$. case strain isolated from the clinical bacteremic infection.

The presented genome showed ANI [27] and is DDH [28] value of $98.4 \%$ and $93.77 \%$ to the strain M40 derived from an environmental source (Table 1). The similarity for the reference genome and the B. casei $\mathrm{S} 18$ strain isolated from the healthy human skin, in turn, was of $98.44 \%$ and $93.8 \%$ respectively (Table 1). However, in comparison with the reference genome of $B$. casei $\mathrm{S} 18$ obtained from the NCBI database, the $\mathrm{S} 51$ strain has additional genes potentially important in its pathogenic potential including antibiotic resistance genes and additional stress response genes: cold shock protein $(\operatorname{Csp} A)$ and heat shock protein $(\operatorname{dnaK})$.

The detection of the beta-lactamase blaTEM-116 encoding gene most probably corresponding to the identified phenotypic resistance against penicillin $\mathrm{G}$ has drawn our particular attention. Although $B$. casei has been reported to be uniformly sensitive to tetracycline, gentamicin, glycopeptides, and rifampin $[2,3,5,6,25]$, decreased susceptibility of the species to beta-lactam agents or clindamycin has been described in previous publications $[2,6,26]$.

Nucleotide sequence accession number This whole-genome NGS project for the S51strain has been deposited at EMBL/GenBank under the accession no. QNGF00000000. The version presented in this article is the first one.

\section{Compliance with ethical standards}

Conflict of interest The authors declare that they have no conflict of interest.

Open Access This article is licensed under a Creative Commons Attribution 4.0 International License, which permits use, sharing, adaptation, distribution and reproduction in any medium or format, as long as you give appropriate credit to the original author(s) and the source, provide a link to the Creative Commons licence, and indicate if changes were made. The images or other third party material in this article are included in the article's Creative Commons licence, unless indicated otherwise in a credit line to the material. If material is not included in the article's Creative Commons licence and your intended use is not permitted by statutory regulation or exceeds the permitted use, you will need to obtain permission directly from the copyright holder. To view a copy of this licence, visit http://creativecommons.org/licenses/by/4.0/.

\section{References}

1. Leal SM Jr, Jones M, Gilligan PH (2016) Clinical significance of commensal Gram-positive rods routinely isolated from patient samples. J Clin Microbiol 54:2928-2936
2. Funke G, Pünter V, Von Graevenitz A (1996) Antimicrobial susceptibility patterns of some recently established coryneform bacteria. Antimicrobial Agents Chemother 40:28742878

3. Bal ZS, Sen S, Karapinar DY, Aydemir S, Vardar F (2015) The first reported catheter-related Brevibacterium casei bloodstream infection in a child with acure leukemia and review of the literature. Braz J Infect Dis 19:213-215

4. Juan CB, Bengoechea AM, Sese BZ, Sánchez MAG (2017) Recurrent endocarditis due to Brevibacterium casei: case presentation and a review of the literature. Enferm Infecc Microbiol Clin 35: 127-136

5. Magi B, Migliorini L, Sansoni A, Cusi MG (2018) Brevibacterium casei bacteremia in a port-a-cath carrier patient:a case report and literature review. Le Infezioni in Medicina 3:263-265

6. Asai N, Suematsu H, Yamada A, Watanabe H, Nishiyama N, Sakanashi D, Kato H, Shiota A, Hagihara M, Koizumi Y, Yamagishi Y, Mikamo H (2019) BMC Infectious Diseases Brevibacterium paucivorans bacteremia: case report and review of literature 19: 344

7. Hoppe-Seyler TS, Jaeger B, Bockelmann W, Geis A, Heller KJ (2007) Molecular identification and differentiation of Brevibacterium species and strains. Syst Appl Microbiol 30:50-57

8. Tatusova T, Ciufo S, Fedorov B, O’Neill K, Tolstoy I (2013) RefSeq microbial genomes database: new representation and annotation strategy. Nucleic Acids Res 42(D1):D553-D559

9. Bankevich A, Nurk S, Antipov D, Gurevich A, Dvorkin M, Kulikov AS, Lesin V, Nikolenko S, Pham S, Prjibelski A, Pyshkin A, Sirotkin A, Vyahhi N, Tesler G, Alekseyev MA, Pevzner PA (2012) SPAdes: a new genome assembly algorithm and its applications to single-cell sequencing. Journal of Comput Biol 19:455-477

10. Aziz RK, Bartels D, Best AA, DeJongh M, Disz T, Edwards RA, Formsma K, Gerdes S, Glass EM, Kubal M, Meyer F, Olsen GJ, Olson R, Osterman AL, Overbeek RA, McNeil LK, Paarmann D, Paczian T, Parrello B, Pusch GD, Reich C, Stevens R, Vassieva O, Vonstein V, Wilke A, Zagnitko O (2008) The RAST server: Rapid Annotations using Subsystems Technology. BMC Genomics 9:75 https://doi.org/10.1186/1471-2164-9-75

11. Zankari E, Hasman $\mathrm{H}$, Cosentino $\mathrm{S}$, Vestergaard $\mathrm{M}$, Rasmussen $\mathrm{S}$, Lund O, Aarestrup FM, Larsen MV (2012) Identification of acquired antimicrobial resistance genes. J Antimicrob Chemother 67:2640-4

12. Alcock et al. (2020). CARD 2020: antibiotic resistome surveillance with the Comprehensive Antibiotic Resistance Database. Nucleic Acids Research, in press. doi: https://doi.org/10.1093/nar/gkz935

13. McCaughey C, Damani NN (1991) Central venous line infection caused by Brevibacterium epidermidis. I infect Secur 23:211-212

14. Lina B, Carlotti A, Leasint V, Devaux Y, Freney J, Fleurette J (1994) Persistent bacteremia due to Brevibacterium species in an immunocompromised patient. Clin Infect Dis 18:484-488

15. Reinert RR, Schnitzler N, Haase G, Lutticken R, Fabry U, Schaal KP, Funke G (1995) Recurrent bacteremia due to Brevibacterium casei in an immunocompromised patient. Eur J Clin Microbiol Infect Dis 14:1082-1085

16. Kaukoranta-Tolvanen SS, Sivonen A, Kostiala AA, Hormila P, Vaara M (1995) Bacteremia caused by Brevibacterium species in an immunocompromised patient. Eur J Clin Microbiol Infect Dis 14:801-804

17. Castagnola E, Conte M, Venzano P, Garaventa A, Viscoli C, Barretta MA et al (1997) Broviac catheter-related bacteraemias due to unusual pathogens in children with cancer: case reports with literature review. J Inf Secur 34:215-218

18. Brazzola P, Zbinden R, Rudin C, Schaad UB, Heininger U (2000) Brevibacterium casei in a 18-year-old female with AIDS. J Clin Microbiol 38:3513-3514 
19. Oğünc D, Gültekin M, Colak D, Timurağaoğlu A, Ongüt G, Mutlu $\mathrm{G}$ et al (2002) Bacteremia caused by Brevibacterium species in a patient with chronic lymphocytic leukemia. Haematologia (Budap) 32:151-153

20. Janda WM, Tipirneni P, Novak RM (2003) Brevibacterium casei bacteremia and line sepsis in a patient with AIDS. J Inf Secur 46: $61-64$

21. Beukinga I, Rodrigueaz-Villalobos H, Deplano A, Jacobs F, Struelens MJ (2004) Management of long-term catheter-related Brevibacterium bacteraemia. Clin Microbiol Infect 10:465-467

22. Ulrich S, Zbinden R, Pagano M, Fischler M, Speich R (2006) Central venous catheter infection with Brevibacterium sp. in an immunocompetent woman: case report and review of the literature. Infection 34:103-106

23. Vecten M, Gouriet F, Cano A, Raoult D (2016) Brevibacterium massiliense bacteremia. ID Cases 7:25-26

24. Bernard KA, Pacheco AL, Burdz T, Wiebe D, Huynh C, Bonner C, German GJ, Benrier A-M (2016) Brevibacterium massiliense (Roux and Raoult 2009) is a later heterotypic synonym of
Brevibacterium ravenspurgense (Mages, Frodl, Bernard and Funke 2009), using whole-genome sequence analysis as a comparative tool. Int J Syst Evol Microbiol 66:4440-4444

25. Troxler R, Funke G, Von Graevenitz A, Stock I (2001) Natural antibiotic susceptibility of recently established coryneform bacteria. Eur J Clin Microbiol Infect Dis 20:315-323

26. Poesen K, Meeus G, Boudewijns M, Colaert J, Doubel P (2012) Relapsing Brevibacterium casei peritonitis: value of 16S rRNA gene sequencing in accurate species identification. PDI 32:341-344

27. Yoon SH, Ha SM, Lim JM, Kwon SJ, Chun J (2017) A large-scale evaluation of algorithms to calculate average nucleotide identity. Antonie Van Leeuwenhoek 110:1281-1286

28. Meier-Kolthoff JP, Auch AF, Klenk HP, Göker M (2013) Genome sequence-based species delimitation with confidence intervals and improved distance functions. BMC Bioinformatics 14:60

Publisher's note Springer Nature remains neutral with regard to jurisdictional claims in published maps and institutional affiliations. 